\title{
Restoration of Kidney Function after Prolonged (Four Months) Anuria Due to Bilateral Renal Artery Occlusion
}

\author{
Abdul-Rahman A El-Magzoub ${ }^{a^{*}}$, Sarra Elamin ${ }^{\mathrm{b}}$ \\ a. MRCP, MSc nephrology (Sheffield), Ahmed Gasim Kidney Transplant Center, Khartoum, Sudan \\ b. MRCP, Ahmed Gasim Kidney Transplant Center, Khartoum, Sudan
}

\begin{abstract}
Introduction: Atherosclerotic renal artery stenosis (RAS) is usually one manifestation of wide spread atherosclerotic disease and its presence has a grave impact on prognosis. RAS is a progressive condition that can remain asymptomatic for a long time, only to present later with accelerated hypertension or renal insufficiency. The first approach to the management of atherosclerotic RAS consists of medical therapy to control blood pressure and other atherosclerotic risk factors. However, surgical or percutaneous revascularization can be of substantial benefit in selected patient groups.
\end{abstract}

Case Report: A 37 year old man who was a heavy smoker and known to have hypertension for the previous five years presented with acute anterior myocardial infarction (MI). His serum creatinine was $8.5 \mathrm{mg} / \mathrm{dl}$ and he needed a session of acute peritoneal dialysis to relieve severe pulmonary edema. Subsequent investigations revealed the presence of bilateral total occlusion of the renal arteries resulting in severe renal impairment and dialysis dependency. He underwent two unsuccessful attempts at angioplasty. Surgical revascularization of both renal arteries was eventually carried out after four months of anuria, and was immediately followed by dramatic improvement of his kidney function. The patient, however, continued to receive antihypertensive medication for adequate blood pressure control.

Conclusion: This case reflects the ability of the kidney to adapt to chronic ischemia, and underscores the importance of considering renal revascularization in all patients with renal artery occlusion, even after long periods of kidney non-functioning.

\footnotetext{
* Corresponding author; Ahmed Gasim Kidney Transplant Center PO Box 992, Khartoum North, Sudan

Email: aelmagzoub@yahoo.com
}

Key words: renal artery occlusion, renaovascular disease, renal revascularization, ischemic nephropathy

\section{Introduction}

Renal arterial disease discovered incidentally can pose no problem for either kidney function or blood pressure control for several years, and as long as blood pressure and kidney function are well maintained, expectant management appears to be entirely appropriate. Nevertheless, revascularization of RAS is often considered with the prospect of improving blood pressure control or the impaired kidney function.

The outcomes of revascularization are heterogeneous. Overall, among RAS patients who underwent percutaneous transluminal renal angioplasty (PTRA) with stenting, 26\% had an improvement in glomerular filtration rate (GFR), 48\% had a stable GFR, whereas $26 \%$ had a decline in GFR [1]. Surgical revascularization has a higher complication rate and higher thirty-day mortality than PTRA (9\% versus 2\%) [2].

The potential for serious deterioration in kidney function after revascularization, be it percutaneous or surgical, and the possibility of serious complications underscore the need to select patients carefully for vascular procedures in the kidney.

\section{Case Report}

A 37 years old man presented to a local cardiac center in December 2004 with an acute anterior myocardial infarction (MI). He was a heavy smoker and had hypertension for five years. He had severe pulmonary edema out of proportion to the degree of his left ventricular dysfunction, and his serum creatinine was found to be $8.5 \mathrm{mg} / \mathrm{dl}$. His pulmonary edema was relieved by an emergent session of peritoneal dialysis, and he was then referred to our center for further management. 
Figure 1: An image of the patient's aortogram, revealing failure of the contrast material to flush the renal artery on either side, or to visualize either kidney

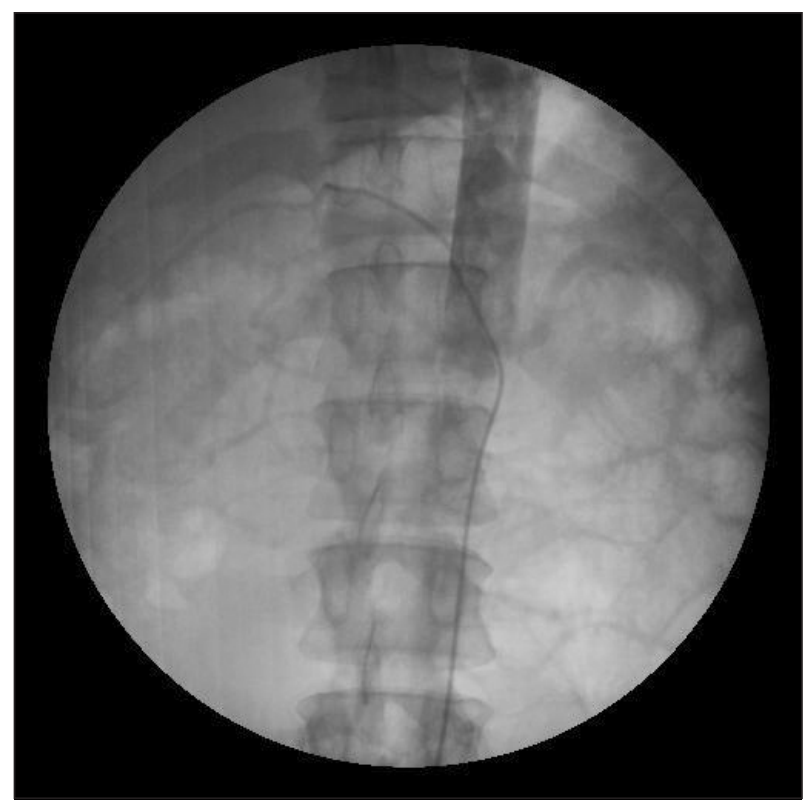

When he first presented to our center, the patient had improved and was asymptomatic. His blood pressure was $130 / 90 \mathrm{mmHg}$ on antihypertensive medication, and his physical examination was unremarkable apart from grade II hypertensive retinopathy.

Investigations revealed a serum creatinine level of $9.5 \mathrm{mg} / \mathrm{dl}$ with normal electrolytes and bland urinary sediment; his hemoglobin was $14.5 \mathrm{~g} / \mathrm{dl}$; he had high lipid profile; his chest $\mathrm{X}$ ray revealed mild pulmonary congestion and bilateral small pleural effusions; his ECG showed evidence of left ventricular hypertrophy and features of recent anterior MI. An abdominal ultrasound scan showed normal sized kidneys with the right kidney being slightly smaller than the left kidney. Renal scintigraphy with Tc ${ }^{99 m}$ DTPA showed that the left kidney had good perfusion with some delay in excretory function and a relative function of $84.5 \%$, while the right kidney had poor perfusion and excretion and a relative function of $15.5 \%$.

The difference in size between the right and left kidneys and the isotope scan findings were suggestive of RAS as a cause of this patient's hypertension; however, we judged his azotemia to be, at least in part, due to a recent episode of severe heart failure and excessive diuresis.

Diuretics were reduced in dose and eventually withdrawn, and he was maintained on low dose aspirin and simvastatin. A small dose of lisinopril (2.5 mg/d) was introduced for blood pressure control and for the well known benefits of angiotensin converting enzyme (ACE) inhibitors on cardiac function.

His renal function improved over several days, and his serum creatinine stabilized at around $2.8 \mathrm{mg} / \mathrm{dl}$. He was then discharged on regular lisinopril $5 \mathrm{mg} / \mathrm{d}$ along with aspirin and simvastatin. He was strongly advised to stop smoking, and informed of the need for regular follow up.

Unfortunately, the patient was lost to follow up for several weeks, during which time he was not taking his medications regularly. He presented again when he became anuric and began to feel unwell, with shortness of breath and excessive fatigue. His blood pressure was $180 / 100 \mathrm{mmHg}$, and he had signs of fluid overload. His serum creatinine had escalated to $30 \mathrm{mg} / \mathrm{dl}$, and this raised the possibility of bilateral renal artery stenosis. Lisinopril was withdrawn, and he was initiated on regular hemodialysis while a renal arteriogram was being arranged. The interventional cardiologist who was to perform the procedure was informed of the possibility of bilateral renal artery stenosis and the need to perform angioplasty with or without stenting if this was the case.

The arteriogram revealed bilateral renal artery occlusion; angioplasty was attempted, but the interventional cardiologist was unable to introduce the catheter into either renal artery (Figure 1).

No collateral supply to the kidneys could be identified on the arteriogram. However, it was judged that the patient may still benefit from revascularization of at least one renal artery. The patient was referred to a vascular surgery center abroad with a CD copy of his renal arteriogram. There, renal arteriography was repeated with a view to another attempt at angioplasty. The second arteriogram confirmed the diagnosis of bilateral occlusion of the renal arteries, and did not reveal any collateral supply to the kidneys. Attempts at angioplasty were again unsuccessful. Surgical revascularization was eventually carried out after 4 months of anuria. Local excision was performed on one side with end to end reanastomosis, and an interposition graft was utilized on the other side using the saphenous vein. The surgery was uneventful, and was followed by rapid improvement in the patient's urine output and kidney function. He was weaned from dialysis, and within a few days of surgery his serum creatinine was $1.3 \mathrm{mg} / \mathrm{dl}$. However, the patient still needed antihypertensive medication for control of his blood pressure.

A coronary angiogram done at the time of renal arteriography revealed occlusion of the circumflex coronary artery, and a peripheral angiography revealed concomitant peripheral vascular disease. In an attempt 
to combat this wide spread atherosclerosis, he was strongly advised to stop smoking and informed about the importance of adequate blood pressure control. He was maintained on Atenolol, Aspirin and Fluvastatin.

Unfortunately, the patient was again lost to follow up for several months, during which time he stopped taking his lipid lowering agent and continued to smoke. He presented again in August 2007, when he experienced recurrent episodes of chest pain and felt short of breath on moderate exertion. He was found to have heart failure (NYHA stage II) with a high blood pressure of 180/110 $\mathrm{mmHg}$.

A chest X ray revealed cardiomegaly and pulmonary congestion, and an echocardiogram revealed global hypokinesia with a left ventricular ejection fraction of $35 \%$. His serum creatinine was $1.6 \mathrm{mg} / \mathrm{dl}$.

\section{Discussion}

The difference in kidney size revealed by the initial ultrasound scan of this hypertensive patient who had long history of smoking and a recent acute coronary event was suggestive of unilateral RAS. This diagnosis was supported, although not confirmed, by the findings of the DTPA scan; however, such scans are frequently difficult to interpret in the setting of renal impairment. Doppler study was not performed because an experienced operator was not available for us at the time.

Although renal revascularization may be considered in the management of RAS associated with hypertension and renal insufficiency, even if it is unilateral, a decision was made to manage this patient conservatively. We suspected that his renal impairment can be, at least in part, a consequence of his recent coronary event and excessive diuresis.

An ACE inhibitor was initiated, both to control his blood pressure and improve his left ventricular function. ACE inhibitors and angiotensin receptor blockers were reported to be effective in treating $86-92 \%$ of cases of hypertension and atherosclerotic RAS [3]. As a result of the wider use of these agents for the treatment of hypertension, it is likely that many individuals with renovascular hypertension are never detected but are effectively treated.

In addition, clinical data suggest that the survival of patients with renovascular hypertension is better when ACE inhibitors are part of therapy than when they are not [4]. This benefit may be in part due to the fact that ACE inhibitors reduce morbidity and mortality in congestive heart failure [5], which is a common co-morbidity in patients with RAS.
Nevertheless, a major concern regarding the use of ACE inhibitors for renovascular hypertension is their potential to cause functional acute renal failure. A rise in serum creatinine of up to $30 \%$ above baseline following the initiation of ACE inhibitors in patients with heart failure is a normal hemodynamic response, and a strong association exists between this acute increase in serum creatinine and long term preservation of renal function. Withdrawal of the ACE inhibitor in such patients should be considered only when the rise in serum creatinine level exceeds $30 \%$ above baseline within the first 2 months of ACE inhibitor initiation, or if hyperkalemia develops [6].

Despite the initial improvement in this patient's renal function, his serum creatinine level rose over the ensuing weeks while taking lisinopril and this raised the suspicion of bilateral RAS [7]. Such an ACE inhibitor associated rise in serum creatinine is usually reversible upon withdrawal of the medication; however, this patient failed to respond to this first line approach.

Renal arteriography to confirm the diagnosis of bilateral RAS was arranged with a view to renal angioplasty. Unfortunately, arteriography revealed bilateral total renal artery occlusion, and attempts at angioplasty were unsuccessful.

Angioplasty has been reported to successfully salvage renal function after bilateral occlusion of the renal arteries by revascularization of one [8-10] or both [11,12] occluded vessels. Percutaneous revascularization should always be attempted first because it is associated with a lower risk of complications compared with surgical revascularization [13], which can still be attempted in case of unsuccessful angioplasty [5].

Unfortunately, several weeks of anuria had already been behind when the diagnosis was confirmed. This raised the question of whether surgical revascularization would benefit the patient, given that transferring the patient to a center equipped with the facilities and experience to perform surgical revascularization entails an extended delay.

Reversing the loss of kidney function with an occlusive disease of the renal arteries poses a major clinical challenge. A number of factors were explored for predicting which patient is most likely to benefit from revascularization. A shrunken kidney, a kidney with cortical necrosis or a kidney proven by biopsy to have irreversible damage is not likely to benefit from revascularization. In addition, a duplex ultrasound scan revealing a renal resistance index of greater than 0.8 indicates small vessel and large vessel disease; such findings predict a poor response to either percutaneous or surgical revascularization with respect to 
improvement in hypertension, renal function, or kidney survival [14].

This patient's kidneys were on the lower side of the normal range of volume estimates, and a renal biopsy was considered unsafe in his condition. Also, although his renovascular disease was most likely a chronic problem, no collateral renal blood supply or nephrogram could be identified on his renal arteriogram. However, the patient agreed to undergo surgical revascularization in impulsive attempt to restore his kidney function. Surgery was eventually carried out after 4 month of anuria. Unpredictably, this was followed by immediate diuresis, and the patient's serum creatinine dropped to 1.3 $\mathrm{mg} / \mathrm{dl}$ within few days of surgery.

Such a rewarding outcome manifests the ability of the kidney to adapt to chronic ischemia, and underscores the importance of considering revascularization in all patients with renal artery occlusion, even after a long period of non-functioning. This is supported by a number of reported cases in which renal dysfunction resulting from bilateral renal artery occlusion was successfully reversed by revascularization, even after prolonged periods of anuria. Unilateral revascularization was successfully used to salvage renal function in 3 elderly chronic renal failure patients who had bilateral renal artery occlusion or diffuse stenosis after being dialysis dependent for 2 weeks, 3 weeks, and 2 months. Two of those patients returned to essentially normal renal function and all had better blood pressure control [15]. Renal revascularization was also successful in restoring kidney function for a young lady who developed total occlusion of the renal artery supplying a solitary functioning kidney as a result of fibromuscular dysplasia; she regained normal renal function and normal blood pressure after being anuric for 31 days [16]. Another young man underwent successful revascularization of an occluded renal artery supplying a solitary functioning kidney after being dialysis dependent for more than six months; this was followed by dramatic improvement in renal function, but he continued to have severe hypertension that was controlled by medication [17].

The ability of the kidney to adapt to chronic ischemia is also manifest by the case of an 11 month old child, who developed renal artery occlusion and anuric renal failure following an unsuccessful attempt at PTRA for a solitary kidney. Despite the prolonged period of anuria, kidney viability was suspected, based on preservation of kidney length and the absence of glomerulosclerosis on kidney biopsy. At 19 months of age, revascularization of the kidney was performed, followed by gradual improvement in renal function so that the child eventually became dialysis-independent [18].
The benefits of revascularization for this patient were not limited to restoration of kidney function and improved blood pressure control, but extended to an improvement in his heart failure symptoms. Such benefit has been previously reported in the literature [19]. The poor cardiovascular status in which this patient presented several months after revascularization underscores the importance of a holistic approach to the management of such patients. Failure of this patient to adhere to lipid lowering therapy and to quit smoking, accompanied by poor blood pressure control, resulted in deteriorating left ventricular function and severe morbidity. This occurred despite a well maintained kidney function.

\section{Conclusion}

This case reflects the ability of the kidney to adapt to chronic ischemia, and underscores the importance of considering renal revascularization in all patients with renal artery occlusion, even after long periods of kidney non-functioning.

\section{References}

1. Isles CG, Robertson S, Hill D. Management of renovascular disease: a review of renal artery stenting in ten studies. QJM. 1999 Mar;92(3):159-67.

2. Alhadad A, Ahle M, Ivancev K, Gottsäter A, Lindblad B. Percutaneous transluminal renal angioplasty (PTRA) and surgical revascularisation in renovascular disease: a retrospective comparison of results, complications, and mortality. Eur J Vasc Endovasc Surg. 2004 Feb;27(2):151-6.

3. Textor SC. ACE inhibitors in renovascular hypertension. Cardiovasc Drugs Ther. 1990 Feb;4(1):22935 .

4. Losito A, Gaburri M, Errico R, Parente B, Cao PG. Survival of patients with renovascular disease and ACE inhibition. Clin Nephrol. 1999 Dec;52(6):339-43.

5. Packer M, Poole-Wilson PA, Armstrong PW, Cleland JG, Horowitz JD, Massie BM, Ryden L, Thygesen K, Uretsky BF. Comparative effects of low and high doses of the angiotensin-converting enzyme inhibitor, lisinopril on morbidity and mortality in chronic heart failure. ATLAS study group. Circulation. 1999 Dec 7;100(23):2312-8.

6. Bakris GL and Weir MR. Angiotensin-converting enzyme inhibitor-associated elevations in serum creatinine: is this a cause for concern? Arch Intern Med. 2000 Mar 13;160(5):685-93. 
7. Schoolwerth AC, Sica DA, Ballermann BJ, Wilcox $\mathrm{CS}$; Council on the Kidney in Cardiovascular Disease and the Council for High Blood Pressure Research of the American Heart Association. Renal considerations in angiotensin converting enzyme inhibitor therapy: a statement for healthcare professionals from the Council on the Kidney in Cardiovascular Disease and the Council for High Blood Pressure Research of the American Heart Association. Circulation. 2001 Oct;104(16):1985-91.

8. Schneider JR, Wright A, Mitchell RS. Successful percutaneous balloon catheter treatment of renal artery occlusion and anuria. Ann Vasc Surg. 1992 Nov;6(6).

9. Sezer M, Nisanci Y, Ozsaruhan O, Olcay A, Ecder T. Stenting for bilateral renal artery occlusion: a report of two cases. Nephrol Dial Transplant. 2003 Dec;18(12):2663-4.

10. Rehan A, Almanaseer Y, Desai DM, Ali A, Yamasaki H. Complete resolution of acute renal failure after left renal artery angioplasty and stent placement for total renal artery occlusion. Cardiology. 2007;108(1):51-4.

11. Fernandez-Fresnedo G, Piña T, Mateos Chaparro F, Sanz de Castro S, González-Tutor A, Bustamante M, de Francisco AL, Arias M. Revascularization of total renal artery occlusion by angioplasty and stent placement. Nefrologia. 2005;25(4):434-7.

12. Han BG, Kim JY, Choi JU, Lee SH, Choi SO. An acute renal failure patient successfully stented for bilateral renal artery occlusion with a distal embolism protection device. Nephrol Dial Transplant. 2004 Jun;19(6):1662-3.
13. Ramos F, Kotliar C, Alvarez D, Baglivo H, Rafaelle P, Londero H, Sánchez R, Wilcox CS. Renal function and outcome of PTRA and stenting for atherosclerotic renal artery stenosis. Kidney Int. 2003 Jan;63(1):276-82.

14. Radermacher J, Weinkove R, Haller H. Techniques for predicting a favourable response to renal angioplasty in patients with renovascular disease. Curr Opin Nephrol Hypertens. 2001 Nov;10(6):799-805.

15. Ascer E, Gennaro M, Rogers D. Unilateral renal artery revascularization can salvage renal function and terminate dialysis in selected patients with uremia. J Vasc Surg. 1993 Dec;18(6):1012-8.

16. Perona PG, Baker WH, Fresco R, Hano JE. Successful revascularization of an occluded renal artery after prolonged anuria. J Vasc Surg. 1989 Jun;9(6):817-21.

17. Cohen DL, Townsend RR, Kobrin S, Genega EM, Tomaszewski JE, Fairman R. Dramatic recovery of renal function after 6 months of dialysis dependence following surgical correction of total renal artery occlusion in a solitary functioning kidney. Am J Kidney Dis. 2001 Jan;37(1):E7.

18. Patterson LT, Bock GH, Guzzetta PC, Ruley EJ. Restoration of kidney function after prolonged renal artery occlusion. Pediat Nephrol. 1990 Mar;4(2):163-5.

19. Messina LM, Zelenock GB, Yao KA, Stanley JC. Renal revascularization for recurrent pulmonary edema in patients with poorly controlled hypertension and renal insufficiency: a distinct subgroup of patients with arteriosclerotic renal artery occlusive disease. J Vasc Surg. 1992 Jan;15(1):73-82. 